\title{
Rational Approximation to $|x|$ at Logarithmic Nodes
}

\author{
Jiao Fang, Yi Zhao, Guojing Hai \\ Department of Mathematics, Hangzhou Normal University, Hangzhou, China \\ Email: China zhaoyi@hznu.edu.cn
}

How to cite this paper: Fang, J., Zhao, Y. and Hai, G.J. (2021) Rational Approximation to $|x|$ at Logarithmic Nodes. Advances in Pure Mathematics, 11, 19-26. https://doi.org/10.4236/apm.2021.111003

Received: December 7, 2020

Accepted: January 18, 2021

Published: January 21, 2021

Copyright $\odot 2021$ by author(s) and Scientific Research Publishing Inc. This work is licensed under the Creative Commons Attribution International License (CC BY 4.0).

http://creativecommons.org/licenses/by/4.0/ (c) (i) Open Access

\section{Abstract \\ Based on a node group $X=\left\{x_{k}=\log \left(\frac{n+k}{n}\right)\right\}_{k=1}^{n}, k=1,2, \cdots, n$, the Newman} type rational operator is constructed in the paper. The convergence rate of approximation to a class of non-smooth functions is discussed, which is $O\left(\frac{1}{n \log (n)}\right)$ regarding to $X$. Moreover, if the operator is constructed based on further subdivision nodes, the convergence rate is $O\left(\frac{1}{n^{2} \log (n)}\right)$. The result in this paper is superior to the approximation results based on equidistant nodes, Chebyshev nodes of the first kind and Chebyshev nodes of the second kind.

\section{Keywords}

Newman Type Rational Operator, Logarithmic, Nodes, Approximation Order

\section{Introduction}

In approximation theory, rational approximation to $|x|$ plays an important role. The approximation to non-smooth functions $|x|$ began with Bernstein's polynomial approximation [1]. In 1964, Newman [2] constructed rational functions $r_{n}(x)$ and approximated $|x|$, with the constructed node set

$$
X=\left\{-a,-a^{2}, \cdots,-a^{n-1}, 0, a^{n-1}, \cdots, a^{2}, a\right\}, a=\exp \left(-n^{-\frac{1}{2}}\right), n=1,2, \cdots,
$$

and the correspondingly rational function to set $X$. It was found that the approximation effect is much better than polynomial approximation. 
After that, scholars have studied the convergence rate of $|x|$ by operators based on different node groups. In 1997, Brutman and Passow [3] considered the zeros of Chebyshev polynomial $T_{2 n}(x)=\cos (2 n \arccos x)$ in interval $(0,1]$, namely, $\left\{x_{k}=\cos \left(\frac{(2 k-1) \pi}{4 n}\right)\right\}_{k=1}^{n}$, or

$$
T=\left\{x_{k}=\sin \left(\frac{(2 k-1) \pi}{4 n}\right)=\cos \left(\frac{(2 n-2 k+1) \pi}{4 n}\right)\right\}_{k=1}^{n},
$$

and investigated the rational interpolation problem of $|x|$. The order of approximation was $O\left(\frac{1}{n \log (n)}\right)$.

In 2010, Huiming Zhang [4] constructed Newman- $\alpha$ type rational operators and the zeros of the second kind of Chebyshev polynomials

$U_{2 n-1}(x)=\frac{\sin (2 n \arccos x)}{\sqrt{1-x^{2}}}$ in interval $[-1,1]$ were considered on the rational interpolation of $|x|, \quad X=\left\{\cos \left(\frac{k \pi}{2 n}\right)=\sin \left(\frac{(n-k) \pi}{2 n}\right)\right\}_{k=1}^{n-1}$, namely, $U(x)=\left\{x_{k}=\sin \left(\frac{k \pi}{2 n}\right)=\cos \left(\frac{(n-k) \pi}{2 n}\right)\right\}_{k=1}^{n-1}$. The order of approximation was $O\left(\frac{1}{n \log (n)}\right)$.

In reference [5], a new set of interpolating nodes was constructed, and the Newman type rational interpolation operator was used to approximate the function $|x|$, and the order of approximation was $O\left(\mathrm{e}^{-\frac{2}{1+\mathrm{e}} \sqrt{n}}\right)$, where $\varepsilon=\sqrt{\frac{3}{2 \sqrt{n}}+\frac{1}{n}}$. In reference [6], the rational operator pairs on a group of encrypted Newman nodes $N=\left\{\mathrm{e}^{-\frac{k}{\sqrt{n}}}\right\}_{k=1}^{2 n}$ approximated $|x|$, and the order of approximation was $O\left(\mathrm{e}^{-2 \sqrt{n}}\right)$.

The approximation $|x|$ of different operators based on different nodes has a wide past. Among these node groups, the zeros of polynomials and exponential type nodes have been considered. It is natural to ask how do the logarithmic nodes perform in the approximation problem.

In this paper, we focus on the convergence rate of $|x|$ at logarithmic nodes $X:=\left\{x_{k}:=\log \left(\frac{n+k}{n}\right)\right\}_{k=1}^{n}$. We prove that, with the node group $X$, the convergence rate of approximation is $O\left(\frac{1}{n \log (n)}\right)$. Furthermore, if the operator 
is constructed based on further subdivision nodes, the convergence rate is improved to $O\left(\frac{1}{n^{2} \log (n)}\right)$. This result reveals the essential to some extent: the more dense the nodes near zero, the better rate of convergence, which also explains the results in some former research, see for example, reference [3].

The Newman type rational operator is constructed in this paper. The Newman type rational operator is defined as

$$
r_{n}(X ; x)=x \frac{p_{n}(x)-p_{n}(-x)}{p_{n}(x)+p_{n}(-x)}, p_{n}(x)=\prod_{k=1}^{n}\left(x_{k}+x\right),
$$

and $h_{n}(X ; x)=\frac{p_{n}(-x)}{p_{n}(x)}$.

\section{Auxiliarily Lemmas}

To verify the desired result, we shall need the following auxiliary results.

Lemma 1. For $x \in N_{+}$, the inequality $\mathrm{e}^{\frac{1}{2}} \leq\left(1+\frac{1}{x}\right)^{x}$ holds true.

Proof. Notice that the function $f(x)=\left(1+\frac{1}{x}\right)^{x}$ is monotonically increasing for $x>1$. Then, $f(1)=2$ is the minimum value and $f(1)>\mathrm{e}^{\frac{1}{2}}$. It is easy to observe that

$$
\left(1+\frac{1}{x}\right)^{x} \geq \mathrm{e}^{\frac{1}{2}}
$$

Lemma 2. For $x, y \in(1, \mathrm{e})$, the inequality $\frac{\log (x)}{\log (y)} \leq \frac{x}{y}$ holds true.

Proof. Write $f(x)=\frac{\log (x)}{x}$. Take the derivative of $f(x)$, we have

$$
f^{\prime}(x)=\frac{1}{x^{2}}-\frac{\log (x)}{x^{2}}
$$

The function $f(x)=\frac{\log (x)}{x}$ is monotonically increasing for $x \in(1, \mathrm{e})$. So the inequality $\frac{\log (x)}{x}<\frac{\log (y)}{y} \Rightarrow \frac{\log (x)}{\log (y)} \leq \frac{x}{y}$ holds true for $x<y$.

Lemma 3. [7] For $0 \leq x \leq \frac{1}{2}$, the inequality $\frac{1-x}{1+x}>3^{-2 x}$ holds true.

Lemma 4. For $x \in[0,1]$, the inequality $\frac{x}{2} \leq \log (x+1) \leq x$ holds true.

Proof. Take the derivative of $f(x)=\log (x+1)-\frac{x}{2}$, we obtain

$$
f^{\prime}(x)=\frac{1}{x+1}-\frac{1}{2}
$$

The function $f(x)$ is monotonically increasing for $x \in[0,1]$ and 
$f(0)=0$. Thus the inequality $\frac{x}{2} \leq \log (x+1)$ holds true for $x \in[0,1]$.

Similarly, for function $g(x)=x-\log (x+1)$, the inequality $\log (x+1) \leq x$ holds true when $x \in[0,1]$.

Lemma 5. [8] For $x \in N_{+}$, the inequality $\log (n)<\sum_{k=1}^{n}\left(\frac{1}{k}\right)$ holds true.

\section{Main Results and Proofs}

Theorem 1. Based on a node group $X=\left\{x_{k}=\log \left(\frac{n+k}{n}\right)\right\}_{k=1}^{n}$, we have

$$
\left|E_{n}(X ; x)\right|=|| x\left|-r_{2 n-1}(X ; x)\right|=O\left(\frac{1}{n \log (n)}\right) .
$$

Proof of Theorem 1. Since $r_{n}(X ; x)=x \frac{p_{n}(x)-p_{n}(-x)}{p_{n}(x)+p_{n}(-x)}$ and $|x|$ are even function, we only need to consider the approximation on the interval $[0,1]$. The proof will be divided into the following cases.

Case 1. $x \in\left[0, \log \left(\frac{n+1}{n}\right)\right]$. Considering

$$
A_{n}=\sum_{k=1}^{n} \frac{1}{\log \left(\frac{n+k}{n}\right)},
$$

and

$$
A_{n}=\sum_{k=1}^{n} \frac{1}{\log \left(\frac{n+k}{n}\right)} \geq \sum_{k=1}^{n} \frac{n}{k} \geq n \log (n) .
$$

Then, we see

$$
\left|h_{n}(X ; x)\right|=\left|\frac{p_{n}(-x)}{p_{n}(x)}\right|=\left|\prod_{k=1}^{n} \frac{x_{k}-x}{x_{k}+x}\right|=\left|\prod_{k=1}^{n} \frac{1-\frac{x}{x_{k}}}{1+\frac{x}{x_{k}}}\right| \leq \mathrm{e}^{-2 x \sum_{k=1}^{n} \frac{1}{x_{k}}} .
$$

Therefore,

$$
\begin{aligned}
\left|E_{n}(X ; x)\right| & =\left|x-r_{n}(X ; x)\right|=\left|2 x \frac{p_{n}(-x)}{p_{n}(-x)+p_{n}(x)}\right| \\
& =\frac{|2 x|\left|\frac{p_{n}(-x)}{p_{n}(x)}\right|}{1+\left|\frac{p_{n}(-x)}{p_{n}(x)}\right|} \leq 2 x \frac{\left|h_{n}(X ; x)\right|}{1-\left|h_{n}(X ; x)\right|} \\
& =2 x\left|h_{n}(X ; x)\right|=2 x \frac{1}{\mathrm{e}^{2 \times A_{n}}} \leq \frac{x}{x A_{n}} \leq \frac{1}{n \log (n)},
\end{aligned}
$$

it is clear that the function achieves its maximum value in the interval 
$\left[0, \log \left(\frac{n+1}{n}\right)\right]$ at $x=\frac{1}{n \log (n)}$.

Case 2. $x \in\left[\log \left(\frac{n+1}{n}\right), \log 2\right]$. We can see

$$
\begin{aligned}
\left|h_{n}(X ; x)\right| & =\left|\frac{p_{n}(-x)}{p_{n}(x)}\right|=\left|\prod_{k=1}^{n} \frac{x_{k}-x}{x_{k}+x}\right| \\
& =\prod_{k=1}^{n} \frac{\log 2-\log \left(\frac{n+k}{n}\right)}{\log 2+\log \left(\frac{n+k}{n}\right)} \leq \prod_{k=1}^{n} \frac{\log \left(2 \frac{n}{n+k}\right)}{\log \left(2 \frac{n+k}{n}\right)} \\
& \leq \prod_{k=1}^{n}\left(\frac{n}{n+k}\right)^{2}=\left\{\left(1+\frac{1}{n}\right)\left(1+\frac{2}{n}\right) \cdots\left(1+\frac{n}{n}\right)\right\}^{-2} .
\end{aligned}
$$

Applying the lemma 1,

$$
\left|h_{n}(X ; x)\right| \leq\left(\mathrm{e}^{\frac{1}{2 n}} \mathrm{e}^{\frac{2}{2 n}} \cdots \mathrm{e}^{\frac{n}{2 n}}\right)^{-2}=\mathrm{e}^{-\frac{n(n+1)}{2 n}}=\mathrm{e}^{-\frac{n+1}{2}} .
$$

Therefore,

$$
\left|E_{n}(X ; x)\right|=\left|x-r_{n}(X ; x)\right|=\left|2 x \frac{p_{n}(-x)}{p_{n}(-x)+p_{n}(x)}\right| \leq 2 x\left|h_{n}(X ; x)\right| \leq 2 \mathrm{e}^{-\frac{n+1}{2}} .
$$

Case 3. $x \in[\log 2,1]$. Noting that

$$
\left|h_{n}(X ; x)\right|=\left|\frac{p_{n}(-x)}{p_{n}(x)}\right|=\left|\prod_{k=1}^{n} \frac{x_{k}-x}{x_{k}+x}\right|=\prod_{k=1}^{n} \frac{\log \mathrm{e}-\log 2}{\log \mathrm{e}+\log 2} \leq \prod_{k=1}^{n} \frac{\log \left(\frac{\mathrm{e}}{2}\right)}{\log (2 \mathrm{e})} \leq 4^{-n} .
$$

Thus we have

$$
\left|E_{n}(X ; x)\right|=\left|x-r_{n}(X ; x)\right|=\left|2 x \frac{p_{n}(-x)}{p_{n}(-x)+p_{n}(x)}\right| \leq 2 x\left|h_{n}(X ; x)\right| \leq 4^{-n+\frac{1}{2}} .
$$

Combining three cases completes the proof of Theorem 1.

Theorem 2. Considering $x^{*}=\frac{1}{n \log (n)}$, we have

$$
\left|E_{n}\left(X ; x^{*}\right)\right| \geq \frac{1}{729 n \log (n)} .
$$

Proof. let $n>4$, we can see that

$$
\left|h_{n}\left(X ; x^{*}\right)\right|=\prod_{k=1}^{n} \frac{\log \left(\frac{n+k}{n}\right)-x^{*}}{\log \left(\frac{n+k}{n}\right)+x^{*}}=\prod_{k=1}^{n} \frac{1-\frac{x^{*}}{\log \left(\frac{n+k}{n}\right)}}{1-\frac{x^{*}}{\log \left(\frac{n+k}{n}\right)}} .
$$

Applying Lemma 3, 


$$
\left|h_{n}\left(X ; x^{*}\right)\right| \geq 3^{-2 x^{*} \sum_{k=1}^{n} \frac{1}{x_{k}}}
$$

where

$$
\begin{aligned}
x^{*} \sum_{k=1}^{n} \frac{1}{x_{k}} & =x^{*} \sum_{k=1}^{n} \frac{1}{\log \left(\frac{n+k}{n}\right)} \leq x^{*} \sum_{k=1}^{n} \frac{2 n}{k}=2 n x^{*} \sum_{k=1}^{n} \frac{1}{k} \\
& \leq \frac{2 n}{n \log (n)}\left(1+\frac{1}{2}+\cdots+\frac{1}{n}\right) \leq 3 .
\end{aligned}
$$

Then we obtain,

$$
\begin{aligned}
n \log (n)\left|E_{n}\left(X ; x^{*}\right)\right| & =\frac{2 n \log (n) \cdot \frac{1}{n \log (n)} \cdot h_{n}\left(X ; x^{*}\right)}{1+h_{n}\left(X ; x^{*}\right)} \\
& \geq h_{n}\left(X ; x^{*}\right) \geq 3^{-6}=\frac{1}{729} .
\end{aligned}
$$

This shows that the approximation order in Theorem 1 cannot be improved.

It is noticed that the density near zero is closely related to the approximation order. If the node near the zero point is further subdivided, the following theorem can be obtained.

Theorem 3. Considering inserting n-degree nodes into the interval $\left[0, \log \left(\frac{n+1}{n}\right)\right]$, which is $\left\{\frac{1}{n} \log \left(\frac{n+k}{n}\right)\right\}_{k=1}^{n}$. The order of approximation on the interval $[0,1]$ is $O\left(\frac{1}{n^{2} \log (n)}\right)$ for $k \geq 1$.

Proof. Taking interval $x \in\left[0, \frac{1}{n} \log \left(\frac{n+1}{n}\right)\right]$ as an example. Considering

$$
A_{n}=\sum_{k=1}^{n}\left(\frac{1}{\frac{1}{n} \log \left(\frac{n+k}{n}\right)}+\frac{1}{\log \left(\frac{n+k}{n}\right)}\right) \text {, }
$$

we can see

$$
\begin{aligned}
A_{n} & =\sum_{k=1}^{n}\left(\frac{1}{\frac{1}{n} \log \left(\frac{n+k}{n}\right)}+\frac{1}{\log \left(\frac{n+k}{n}\right)}\right) \geq \sum_{k=1}^{n} \frac{n^{2}}{k}+\sum_{k=1}^{n} \frac{n}{k} \\
& \geq n^{2} \log (n)+n \log (n) \geq n^{2} \log (n) .
\end{aligned}
$$

Direct computation together with (9) result in

$$
\begin{aligned}
\left|E_{n}(X ; x)\right| & =\left|x-r_{n}(X ; x)\right|=\left|2 x \frac{p_{n}(-x)}{p_{n}(-x)+p_{n}(x)}\right| \\
& \leq 2 x\left|h_{n}(X ; x)\right|=2 x \frac{1}{\mathrm{e}^{2 \times A_{n}}} \leq \frac{1}{A_{n}} \leq \frac{1}{n^{2} \log (n)} .
\end{aligned}
$$


The proof on other intervals can refer to theorem 1.

\section{Conclusions}

In this paper, based on the node group $X=\left\{x_{k}=\log \left(\frac{n+k}{n}\right)\right\}_{k=1}^{n}$, we use Newman type rational operators to approximate $|x|$, and derive the approximation order $O\left(\frac{1}{n \log (n)}\right)$. When $r_{n}(x)$ is discussed on the interval $\left[0, \log \left(\frac{n+1}{n}\right)\right]$, the approximation effect is the worst, which is $O\left(\frac{1}{n \log (n)}\right)$; on the interval $\left[\log \left(\frac{n+1}{n}\right), \log 2\right]$, the approximation effect is better, which is $O\left(\mathrm{e}^{-n}\right)$; on the interval $[\log 2,1]$, the approximation effect is the best, it is $O\left(4^{-n}\right)$.

Considering that the higher the density of the node near zero, the better the approximation effect is, we further insert the nodes of degree $n$ to make the approximation order reach $O\left(\frac{1}{n^{2} \log (n)}\right)$. This result is better than $|x|$ on equidistant nodes ([9]), Chebyshev nodes of the first kind ([3]) and Chebyshev nodes of the second kind ([4]).

\section{Acknowledgements}

The work is supported by the Natural Science Foundation of China under grant number 11601110 and China Scholarship Council.

\section{Conflicts of Interest}

The authors declare no conflicts of interest regarding the publication of this paper.

\section{References}

[1] Bernstein, S.N. (1913) Sur la meilleure approximatio $\mathrm{n}$ de $\mathrm{x}$ pardes polynome s de degres donnes. Acta Mathematica, 37, 1-57. https://doi.org/10.1007/BF02401828

[2] Newman, D.J. (1964) Rational Approximation to $|x|$. Michigan Mathematical Journal, 11, 11-14. https://doi.org/10.1307/mmj/1028999029

[3] Brutman, L. and Passow, E. (1997) Rational Interpolation to $|x|$ at the Chebyshev Nodes. Bulletin of the Australian Mathematical Society, 56, 81-86. https://doi.org/10.1017/S0004972700030756

[4] Zhang, H.M. and Li, J.J. (2010) Rational Approximation to $|x|$ at the Chebyshev Nodes of the Second Kind. Journal of Zhengzhou University (Natural Science Edition), 42, 1-3.

[5] Zhan, Q. and Xu, S.S. (2015) The Newman Rational Interpolating Approximation Based on a New Set of Nodes. Journal of Anhui University of Science and Technology (Natural Science), 35, 83-86. 
[6] Zhang, H.M. and Li, J.J. (2018) On Rational Interpolation to $|x|$ at the Dense Newman Nodes. Chinese Journal of Engineering Mathematics, 35, 408-414.

[7] Xu, J.H. and Zhao, Y. (2017) On Rational Interpolation to $|x|$. Acta Scientiarum Naturalium Universitatis Sunyatseni, 56, 64-67.

[8] Nie, W.X. (2016) Harmonic Series Sum(1/k) from $\mathrm{k}=1$ to $\mathrm{n}$ and Its Application. Mathematics Communication, No. 12, 38-40.

[9] Werner, H. (1982) Rationale Interpolation von $|x|$ in Quidistanten Punkten. Mathematische Zeitschrift, 180, 11-17. https://doi.org/10.1007/BF01214996 